\title{
La cuestión por la novedad en el pragmatismo como lógica de la abducción*
}

\author{
The question of novelty in pragmatism \\ as the logic of abduction
}

\section{A questão pela novidade do pragmatismo como lógica da abdução}

Fecha de entrega: 15 de septiembre de 2014 Fecha de evaluación: 20 de noviembre de 2014 Fecha de aprobación: 15 de diciembre de 2014

\section{Manuel Darío Palacio Muñoz}

\section{Resumen}

El presente artículo presenta cuatro visiones sobre la función de la abducción en el pragmatismo de Peirce y el modo en que afronta el tema de la novedad en la ciencia. La primera visión reduce la abducción a una intuición. La segunda indica que la abducción no sirve sin un "instinto descubridor", la tercera apunta a que la abducción es insuficiente para explicar la novedad en la ciencia. La cuarta visión, que es la que se sostiene en este artículo, afirma que desde el pensamiento de Peirce se dan las herramientas suficientes para resolver el problema

* Trabajo resultado de la investigación del autor en el seminario de Pragmatismo orientado por el profesor Andrés Páez en la Universidad de los Andes.

* Estudiante del Doctorado de la Universidad de los Andes. Contacto: md.palacio10@uniandes.edu.co 
de la novedad en la ciencia desde el pragmatismo como lógica de la abducción. Estas herramientas son las tres tesis cotarias.

Palabras clave: Peirce, pragmatismo, abducción, tesis cotarias, novedad

\section{Abstract}

This paper shows four visions about the function of abduction in Peirce's pragmatism and the way to face newness in science. The first view reduces abduction to induction. The second view claims that abduction is useless without any "discoverer instinct". Third view says abduction is insufficient to explain newness on science. Fourth view, which we defend in this paper, asserts that Peirce's philosophy offers enough tools in order to explain how works the pragmatism as abduction's logic over the quest of newness in science. This tools are the cotary propositions.

Keywords: Peirce, pragmatism, abduction, cotary propositions, newness

\section{Resumo}

O presente artigo apresenta quatro visões sobre a função da abdução no pragmatismo de Peirce e o modo em que afronta o tema da novidade da ciência. A primeira visão reduz a abdução a uma intuição. A segunda indica que a abdução não serve sem um "instinto descobridor", a terceira aponta a abdução como suficiente para explicar a novidade da ciência. A quarta visão, que é a que defendemos neste artigo, afirma que, desde o pensamento de Peirce, se dão as ferramentas suficientes para resolver o problema da novidade na ciência, desde o pragmatismo como lógica da abdução. Estas ferramentas são as três teses cotárias.

Palavras-chave: Peirce, pragmatismo, abdução, teses cotárias, novidade.

\section{Introducción}

Una forma de entender el pragmatismo es definirlo en términos de la orientación de la experiencia. Si bien esta consideración aparece velada entre los pensamientos de 
los distintos autores pragmatistas, resulta comprensible en el operar de las premisas pragmatistas. Sin entrar en detalles, la máxima pragmática es un claro ejemplo de esto, pues establece que la concepción de un objeto es la concepción de sus efectos concebibles. De tal manera que un objeto que carece de efectos concebibles no puede ser 'útil' para la orientación de la experiencia.

Esta formulación del pragmatismo enmarca el problema del que parte la presente investigación y que consiste en la pregunta por la novedad en la experiencia. Si bien la concepción de un objeto es la concepción de sus efectos concebibles, ciertamente no todos los efectos concebibles son aquellos que pueden esperarse; o dicho en otros términos, es posible que aparezca un efecto sorpresivo que no se esperaba. ¿Cómo puede el pragmatismo, como lógica de la abducción, guiar la experiencia ante el carácter sorpresivo de un hecho? Esta es la pregunta base que dirige el texto.

Para responderla se procederá en dos grandes pasos. El primero consiste en establecer la vinculación entre pragmatismo y abducción como forma inferencial, en donde la inferencia busca aplacar el carácter sorpresivo de un hecho. El segundo paso consiste en el análisis de ciertas posturas que consideran la versatilidad y algunas implicaciones de la abducción peirceana respecto al problema de la novedad, e incluso del descubrimiento, en la propuesta de Peirce. Revisando las dificultades y omisiones de estas posturas, se propone la tesis del texto, consistente en que la respuesta a la cuestión de la novedad en la abducción debe darse a partir de la vinculación de las tres tesis cotarias como márgenes del pragmatismo, entendido como lógica de la abducción. Finalmente, las conclusiones pertinentes expondrán algunos corolarios de la posición defendida.

\section{Pragmatismo y abducción}

Se ha indicado que el pragmatismo puede entenderse como una forma de orientar la experiencia. Para hacerlo, se desplaza a partir de condiciones antecedentes a posibilidades futuras. ${ }^{1}$ Tal desplazamiento consiste en un proceso inferencial de unas a las otras. De las formas básicas de inferencia, el pragmatismo se identifica directamente con la hipótesis, o como el mismo Peirce la denominará más tarde, la abducción.

1 La formulación está expresada ya por Corrington (An introduction to C.S. Peirce, 1993) al indicar: "As should by now be clear, pragmatism moves away from antecedent contidions toward future possibilities..." 
Tenemos pues que Este tipo de inferencia se llama hacer una hipótesis. Es la inferencia de un caso a partir de una regla y un resultado. ${ }^{2}$

El carácter abductivo del pragmatismo obedece al modo en que se articula la inferencia de la regla, el caso y el resultado. De las particularidades de las relaciones inferenciales entre estos elementos, surgen los distintos modos de inferencia, que Peirce clasifica como inducción, deducción y abducción (hipótesis). La inferencia abductiva, que caracterizará al pragmatismo en términos de Peirce, consiste en la postulación de algo que resulta de una clase diferente a lo observado, y que lo explica. ${ }^{3}$

El conocido ejemplo de Peirce explica la forma del razonamiento en la abducción:

Regla.-Todas las judías de esta bolsa son blancas.

Resultado.-Estas judías son blancas.

Caso.-Estas judías son de esta bolsa. ${ }^{4}$

Mientras que la deducción es una inferencia analítica, las otras dos son sintéticas. Por tanto, el matiz diferenciador entre la inducción y la abducción consiste en la posibilidad ${ }^{5}$ de inferir un caso diferente tanto de la regla como del resultado: Mediante la inducción, concluimos que hechos similares a los hechos observados son verdaderos en casos no examinados. Merced a la hipótesis, concluimos la existencia de un hecho muy diferente de todo lo observado, del cual, según las leyes conocidas, resultaría necesariamente algo observado. ${ }^{6}$

2 "This case of inference is called making an hypothesis. It is the inference of a case from a rule and result". (EP, DIH p. 188-189). Para las citas de Peirce se sigue la traducción de la Universidad de Navarra disponible en el sitio web del Centro de Estudios Peirceanos (http://www.unav.es/gep/Peirce-esp.html). Se indica a pie de página el original en inglés, tomado de la edición de Essentials Peirce.

3 En palabras de Peirce: "Hypothesis is where we find some very curious circumstance, which would be explained by the supposition that it was a case of a certain general rule, and thereupon adopt that supossition" (EP, DIH p. 189)

4 Rule - All the bean from this bag are White. Result - These beans are white. Case - These beans are from this bag. (EP, DIH p. 188-189)

5 Si bien es algo que el mismo Peirce dice, en diversos apartados, la preponderancia que adquiere aquí posibilidad es la de un carácter de probabilidad. (Nubiola, 2005)

6 By induction, we conclude that facts, similar to observed facts, are true in cases not examined. By hypothesis, we conclude the existence of a fact quite different from anything observed. (EP 1.194). 
La abducción está abierta a la posibilidad; por tanto, es falible. Peirce, por ello, considera que es preciso que el proceso de "hacer hipótesis" sea liderado por tres reglas: la primera consiste en que la hipótesis debe estar distintamente puesta como una cuestión. La segunda consiste en no hacer una especial clase de predicciones para las que la hipótesis se sepa suficientemente adecuada. Finalmente, la tercera consideración es que el fracaso, así como el error, debe ser tenido en cuenta para la formulación de nuevas hipótesis. (EP, DIH p. 193). Esta lógica de la abducción será la que en escritos posteriores describa la naturaleza propia del pragmatismo peirceano.

En tales textos posteriores, correspondientes a las lecciones de Harvard, Peirce considera que el pragmatismo se entiende como lógica de la abducción y define su tarea fundamental en el aplacamiento del carácter sorpresivo de un cierto hecho. Así: Se observa el hecho sorprendente C; pero si A fuese verdadero, C sería una cosa corriente, luego hay razones para sospechar que $A$ es verdadero. ${ }^{7}$

Ante tal formulación, Peirce considera que A no puede ser abductivamente inferida hasta que su contenido no esté enteramente presente en las premisas de un modo tal que «si A fuera verdadero, C sería un caso corriente». La consecuencia de esta consideración indica que la abducción resuelve el carácter sorpresivo de $\mathrm{C}$ con la inferencia de A.

La abducción tiene por tarea, entonces, la explicación y la subsiguiente eliminación de la sorpresa ${ }^{8}$ dentro de un entramado inferencial que explique el hecho sorpresivo haciéndolo comprensible y poniéndolo a disposición del saber. En este orden de ideas, la sorpresa surge por una respuesta negativa a ciertas expectativas. No se espera $\mathrm{C}$, pero $\mathrm{C}$ es observado. Aclarar la razón por la que $\mathrm{C}$ es sorpresivo implica la no observación de $\mathrm{A}$, puesto que si A fuera cierto, $\mathrm{C}$ sería un hecho común. Por lo tanto, es explicativamente lógico suponer A, pese a que A no es observado directamente. Es por ello que A es fruto de una inferencia. Ciertamente A tiene un carácter de probabilidad que tiene la posibilidad de ser falsa.

7 The surprising fact, $C$, is observed; But if $A$ were true, $C$ would be a matter of course. Hence, there is reason to suspect that $A$ is true. (EP, PLA)

8 Jaime Nubiola señala que la palabra "surprise" posee alrededor de 120 menciones en el corpus de las obras de Peirce, la mayoria en los textos de 1901. Es por tanto una categoría significativa más que una mera descripción para un hecho inesperado. Cf. (Nubiola, 2005) 
Un evento que puede ser respondido de un modo habitual no causa ninguna sorpresa. Por el contrario, un hecho "sorpresivo" requiere un cambio en nuestro hábito racional de creer; este demanda una explicación. Una explicación hace racionales los hechos, esto es, permite la adquisición de una creencia que explique el hecho, haciéndolo razonable. ${ }^{9}$

Pero el carácter sorpresivo afecta la solidez de una creencia. Así, no es identificable este carácter sorpresivo con una duda al estilo cartesiano: No pretendamos dudar en la filosofía de aquello de lo que no dudamos en nuestros corazones. ${ }^{10}$ De esta manera, podemos identificar este carácter sorpresivo con una duda genuina ${ }^{11}$. Entonces, la manera en que se resuelve tal sorpresa implica la inferencia como una forma de resolver una duda auténtica, esto es, como la fijación de una creencia.

Dentro de los métodos de fijación de la creencia se encuentra, como método privilegiado por el pragmatismo, el método científico. Ya que, los métodos de la tenacidad, de la autoridad y de la introspección (o método a priori), poseen un valor relativo, al no permitir el ejercicio crítico-comunitario de la ciencia. De tal manera, el pragmatismo queda vinculado con el proceder de la ciencia, como teoría de la ciencia. Dentro de esta consideración, Peirce formula el pragmatismo como lógica de la abducción y le asigna una doble función: por un lado, brindar una expedita eliminación de todas las ideas esencialmente no-claras y en segundo lugar, brindar un soporte y ayudar a proveer distinción a las ideas esencialmente claras, pero que son aprehendidas con más o menos dificultad. ${ }^{12}$

Si la abducción resuelve el carácter sorpresivo de un hecho y la función del pragmatismo, como lógica de la abducción, radica en la eliminación de ideas no-claras y en el discernimiento de las que son claras, surge la pregunta de cómo el pragmatismo

9 Traducción propia: An event that can be answered in an habitual form does not cause any surprise. On the contrary, a 'surprising' fact requires a change in our rational habit of belief; it demands an explanation. An explanation makes the facts rational, that is, it enables the acquisition of a belief that explains the fact, rendering it reasonable. (Nubiola, 2005, pág. 124)

10 Let us not pretend to doubt in philosophy what we do not doubt in our hearts (EP, FI p. 65)

11 Esta relación la tomamos de la formulación de Peirce: "For belief, while it lasts, is a strong habit, and as such, forces the man to believe until some surprise breaks up the habit". (CP 5.524)

12 El original reza asi: first place, to give us an expeditious riddance of all ideas essentially unclear. In the second place, it ought to lend support [to] and help to render distinct, ideas essentially clear but more or less difficult of apprehension... (EP, p. 239) [Traducción propia]. 
puede explicar bajo una lógica abductiva la novedad en la ciencia, puesto que en ella, como ampliación del conocimiento, radica el quid de la ciencia moderna. ${ }^{13}$ Esta consideración conduce al siguiente cuestionamiento: ¿cómo explicar la novedad en la ciencia, tanto en los cambios de paradigmas como en los descubrimientos más disciplinares, en la medida en que la tarea del pragmatismo peirceano, como lógica de la abducción, consiste en la eliminación del carácter sorpresivo, mediante la fijación de la creencia y la depuración (aclaración) de las ideas?

\section{La pregunta por la novedad y la abducción}

La ciencia moderna se ha fraguado sobre la idea del aumento del conocimiento. Las grandes críticas de los primeros filósofos y científicos modernos a la lógica y ciencia medieval, consistieron en denunciar que su ciencia no consistía en un aumento del conocimiento sino en una explicación silogística de los hechos del mundo a partir de premisas aceptadas. En sentido estricto, se trataba más de una explicitación del conocimiento que de un incremento del mismo. La novedad científica, como característica de la ciencia moderna, hace parte del quid más propio de la ciencia. ${ }^{14}$

En este sentido, la pregunta por la novedad en la ciencia adquiere el matiz de la pregunta por el descubrimiento. Resulta innegable reconocer el carácter innovador del descubrimiento en la ciencia moderna, a tal punto que ciertas posturas la han limitado a este carácter. La cuestión que se aborda es la siguiente: si el pragmatismo se adhiere a la ciencia en su proceder como en su objeto, cómo podría explicarse abductivamente el descubrimiento y la novedad. Entre las opciones lógicas a la respuesta de esta pregunta está la afirmación y negación de la cuestión. Pero además, existen otras dos formas de ver el problema y que consiste en demostrar que la propuesta del pragmatismo es válida pero insuficiente. De tal manera que pueden figurarse cuatro planteamientos. El que dice que no es posible la novedad desde una propuesta pragmática, el que indica que es posible pero que se necesita superar el

13 Ciertamente en la abducción hay novedad, como bien lo anota (Génova, 1997), pero la cuestión gira en torno a cómo tal novedad puede explicarse en una lógica de fijación de ideas y de aclaración de las ideas, esto es, en el pragmatismo.

14 En el fondo esta es la problemática que ya se había establecido desde Bacon hasta Kant, quien considera que los juicios de la ciencia han de ser sintéticos, para que efectivamente pueda hablarse de novedad en el conocimiento; sin embargo, su carácter debía ser a priori. Esta es la cuestión que subyace a la pregunta acerca de cómo son posibles los juicios sintéticos a priori. 
pragmatismo, una tercera opción afirma que la abducción define la novedad en la ciencia, pero que se precisan de otros elementos, $y$, una última postura que afirma que desde el pragmatismo como lógica de la abducción es posible resolver la novedad.

Por tanto, la pregunta por la novedad en una lógica de la abducción puede entenderse, y se ha entendido, en cuatro direcciones. La primera consiste en una negación que implica la imposibilidad de una novedad científica. La segunda hace referencia a la limitación de la novedad a elementos que van más allá de la abducción como forma de aseguramiento y de eliminación de la sorpresa. Por tanto, la novedad habría que hallarla en un uso heurístico de la abducción. La tercera consideración indica que la abducción en la ciencia, si bien pone en movimiento los mismos mecanismos que la abducción en la vida cotidiana, implica el uso de las otras formas de inferencia (inducción y deducción). Una cuarta vía, consiste en mostrar que efectivamente Peirce, con la definición del pragmatismo como lógica de la abducción, responde directamente a la pregunta por la novedad en la ciencia a partir de la abducción misma. Esta cuarta consideración será la que se sostendrá en el presente texto.

\subsection{La imposibilidad de la novedad en la filosofía de Peirce}

La primera postura que se analiza correspode a la sostenida por el profesor Cristian Soto, en la que se declara la imposibilidad y dificultad inherente a la abducción pragmática de resolver el problema del descubrimiento en la ciencia.

... Peirce al indagar la naturaleza del razonamiento involucrado en la generación de hipótesis, que pueden datarse con claridad en textos de 1878, destaca por su penetración intuitiva; no obstante el mismo Peirce no ha llegado a una concepción definitiva y precisa de la cuestión ni, mucho menos, ha vislumbrado los distintos alcances del problema del descubrimiento. (Soto, 2011)

La negación de encontrar en Peirce una respuesta a la pregunta por la forma abductiva del descubrimiento obedece a una turbia consideración de la tercera tesis cotaria. Así, el profesor Soto considera que puede traducir la palabra insight por intuición. Pese a que él es consciente de la crítica que Peirce establece a la posibilidad de una cognición no condicionada por una anterior asume el término. Pero no es cuestión puramente de traducción, el profesor Soto considera que ciertamente en el contenido 
de la tercera tesis cotaria ya habría un elemento de inmediatez perceptiva que no duda en denominarlo 'intuición'. ${ }^{15}$

Así, la propuesta del profesor Soto consiste en modificar la tercera tesis cotaria, para poder incluir, dentro del pensamiento de Peirce, la categoría de intuición que ya el autor anglosajón había eliminado de su propuesta filosófica desde los primeros escritos. Así, como se observará en el cuarto apartado de este numeral, tal interpretación de la tercera tesis cotaria es la que le impide al profesor Soto entender de una manera diferente el descubrimiento y la novedad dentro de la abducción y le obliga a introducir nuevamente un cierto tipo de intuición.

Por lo demás, para el profesor Soto, la propuesta de Peirce respecto al tema del descubrimiento en la ciencia, apenas posee elementos rescatables, que para mostrar su utilidad, se precisaría de un giro que amplíe la aplicación de los argumentos de Peirce.

\subsection{La abducción complementada por la heurística}

Existe una interpretación usual y en cierta medida, consolidada, que considera que bajo la lógica de la abducción subyace una especie de heurística que asume la abducción como un punto de partida, pero que precisa de algunas potencias extra-inferenciales. Esta es la tesis sostenida por Gorlée (1996), Paavola (en prensa), Gonzalez y Haselager (2005). En realidad, la heurística abductiva es esencial en todos los actos interpretativos: es decir, los actos que requieren un descubrimiento genial de cualquier clase y en todos los campos de investigación, tanto teóricos como prácticos.

Esta postura afirma que la abducción precisa de una especie de instinto para que se pueda guiar, pues ciertamente la abducción, en tanto inferencia sintética, vincula un elemento que no está contenido en la premisa (pues sería una inferencia analítica, esto es, una deducción). Pero no habría forma de definir qué tipo de condiciones determinan el contenido de la inferencia, por lo tanto, este contenido se vería indeterminado por una infinita posibilidad de hipótesis. Para ello, la abducción funcionaría con una especie de "instinto adivinatorio": La abducción consiste en hacer adivinaciones

15 Cf (Soto, Abducción como 'inferencia' y como 'intuición' en Peirce, 2006). Específicamente en la nota 5 se indica la precisión de la traducción, y en ella se afirma la identificación de la abducción con la intuición. En la página 5 del documento se indica expresamente: Como puede verse, no es forzar los planteamientos del filósofo norteamericano sostener que la naturaleza del razonamiento abductivo es dual, involucrando caracteristicas inferenciales e intuitivas. 
inteligentes y relevantes que implican conexiones novedosas entre pregunta y solución a través de un destello de comprensión. (Gorlée, 1996). Por tanto, desde esta postura no se podría hablar en sentido estricto de una lógica de la abducción sino de una heurística de la abducción, ya que no habría forma de establecer las determinaciones y reglas de una inferencia para que se produjera una abducción lógica.

Como se ve, también esta postura obvia la consideración que Peirce hace de la abducción con las tesis cotarias. Estas tres tesis le permiten fundar el carácter lógico de la abducción para que, dentro de ese marco, se generen hipótesis de manera lógica sin recurrir a facetas semi-concientes o instintivas. Eso se trabajará en el apartado 2.4 del presente texto.

\subsection{La abducción como un proceso por complementar}

Callaway (2008) al igual que Génova (1997) afirman que la lógica de la abducción consiste en la formulación de una hipótesis, que se juega en el orden científico como una abducción en la vida personal. Pero en tanto que la abducción permite resolver lo "sorpresivo" de un hecho, la ciencia se ocupa de otros procesos lógicos que complementan la formulación abductiva y que consisten en la inducción y deducción.

En este sentido, la abducción no es independiente de los otros procesos de inferencia que constituyen el operar del método científico. Así, la abducción está directamente relacionada con la manera en que opera la deducción y la inducción:

La investigación comienza por el hecho sorprendente que choca contra los hábitos, el acontecimiento contrario a las expectativas, la ruptura de la creencia (belief) en cuanto que disposición operativa. A partir de aquí, la investigación se desarrolla en tres etapas: abducción, deducción e inducción... La inferencia abductiva propone una hipótesis que debe ser en sí misma verosímil, aceptable, pero no da ninguna otra garantía... En este sentido la abducción, aun siendo el origen de la explicación, es un argumento ineficaz, puesto que no puede por sí misma reformar los hábitos racionales, las creencias, sin el complemento de la deducción y la inducción. (Génova, 1997)

Como ya se ha indicado con antelación, la propuesta de Génova es exacta en el horizonte del funcionamiento de la ciencia. Ciertamente Peirce no considera que dentro de esta (la ciencia), una forma inferencial prime sobre la otra, sino que en la dinámica 
de los distintos modos de inferencia surge el afianzamiento de la creencia, lo que equivale a la resolución del carácter sorpresivo de un determinado hecho. Por tanto, la relación entre la abducción que propone, la deducción que extrae consecuencias y la inducción que aplica y comprueba, conforma la estructura lógica que no solo determina la estructura de la ciencia sino del mismo pensamiento.

La cuestión queda relativamente señalada con esta respuesta, pues la novedad que aparece en la abducción es apenas plausible. Su verdad se muestra en la totalidad del proceso inferencial lógico y en su 'calibración' a partir de sucesivas deducciones, inducciones, abducciones, deducciones, etc. Esto es exactamente lo que Peirce señalaba con la segunda y la tercera regla para formular una hipótesis.

Esta misma idea es sostenida por Callaway (2008):

Para Peirce, la relación del pragmatismo y la abducción se torna en la cuestión de los efectos prácticos concebibles de una hipótesis. Una vez confirmados y establecidos por la investigación científica, es claro que nuestra antigua hipótesis puede, de hecho, implicar efectos prácticos. Aunque la pregunta más interesante es ver cómo la consideración de las consecuencias, metodológicas y concebibles, de una hipótesis alternativa pueden ayudar a dirigir la atención entre ellas, antes de la confirmación o negación de las predicciones competentes. ${ }^{16}$

Confirmation or disconfirmation corresponden a una estancia de validación más allá de la misma inferencia abductiva. Esto significa lo mismo que lo dicho anteriormente, que la hipótesis precisa de algo más allá para cumplir con la función de la inferencia. Ante esta consideración no podría haber, peirceanamente, ninguna objeción.

Sin embargo, en la propuesta que aquí se esgrime, se señala una ausencia de referencia al papel de las tesis cotarias dentro de la formulación del pragmatismo como lógica de la abducción. En este sentido, las tres posturas anteriores adolecen de la vinculación de un soporte teórico y metodológico que Peirce se

16 En original la versión en inglés. Traducción propia: "For Peirce, the relation of pragmatism to abduction turns on the question of the conceivable practical effects of an hypothesis. Once confirmed and established by scientific inquiry, it is clear that our erstwhile hypothesis may indeed embody practical effects. Yet the more interesting question is to see how consideration of conceivable methodological consequences of alternative hypotheses may help direct attention among them, prior to confirmation or disconfirmation of competing predictions." 
esfuerza en repetir en dos de sus conferencias de Harvard (Nature of Meaning / Pragmatism as the Logic of Abduction) con el aparato teórico de la inferencia abductiva. Por tal razón, el siguiente apartado consiste exactamente en señalar este punto. El carácter abductivo del pragmatismo no ha de pensarse por fuera de la delimitación que establecen las tesis cotarias y por tanto la novedad y el descubrimiento científico han de pensarse dentro de la delimitación establecida por estas tesis cotarias.

\subsection{El Pragmatismo como lógica de la abducción y su soporte en las tesis cotarias}

Tras este breve repaso por las consideraciones acerca del carácter abductivo del pragmatismo, resulta pertinente establecer algunos matices que no son tenidos en cuenta por los autores que se han referido hasta ahora. El matiz más importante está en la pertinencia de la enunciación de las tesis cotarias para calificar al pragmatismo como lógica de la abducción. Esto conlleva tres grandes implicaciones de acuerdo a cada una de las tesis cotarias. Antes de ello, conviene exponer las tesis cotarias para establecer su vinculación con la abducción.

La primera tesis cotaria establece la reconocida formulación Nihil est in intellectu quin prius fuerit in sensu. Sin embargo, Peirce rápidamente precisa que la acepción que le quiere dar a esta primera tesis difiere de la tradicional concepción aristotélica. Por intellectus Peirce entiende el significado de cualquier representación en cualquier tipo de cognición, virtual, simbólica o cualquiera que pueda ser (EP, II, 16, PLA p. 226-227). El ejemplo que utiliza para aclarar esta posición es la crítica a Berkeley y a ciertos nominalistas que niegan que se pueda tener una idea de un triángulo en general. Peirce enfatiza que: si tenemos o no tenemos una idea de triángulo en sentido psicológico. Tenemos un intellectus, un significado, del cual es un elemento el triángulo en general. ${ }^{17}$ La posesión de un significado determina el sentido de aquello que está en el entendimiento, más que la mera posesión psicológica y atómica de una idea determinada. En este mismo orden, Peirce propone su propia acepción para el término

17 Whether we have an idea of a triangle in some psychological sense or not, I do not, as logician, care. We have an intellectus, a meaning, of which the triangle in general is an element. (EP. PLA) 
sensu, con el cual Peirce significa "Juicio perceptual" (perceptual judgment). ${ }^{18}$ En definitiva, la primera tesis cotaria indica que no hay significaciones atómicas que se capten intuitivamente de la experiencia y se consoliden en ideas separadas, sino que todo aquello que hay en el entendimiento ha sido captado inferencialmente desde la experiencia misma.

La segunda tesis cotaria es la explicitación del enunciado del sensu, esto es, que los juicios perceptuales contienen elementos generales, de tal modo que proposiciones universales pueden deducirse de estos juicios. La explicación de esta segunda tesis se encuentra en la conferencia anterior (EP, II, 15, NM, p. 223), a propósito del ejemplo del perro. Peirce señala que los juicios perceptuales de los perceptos de perros tienen varios elementos generales y que estos han sido generalizados por abducción, principalmente, con algunas pequeñas dosis de inducción. En el fondo, lo que se está jugando aquí es el problema filosófico de definir en qué consiste lo existente, si se trata de un cúmulo de objetos atómicos o si es un entramado de relaciones. ${ }^{19}$ Podría ponerse el ejemplo de un libro. En primera instancia, y muy al estilo de Berkeley, se podría hablar de la idea de libro. Sin embargo, si al libro que está sobre la mesa se le quita una hoja, sigue siendo un libro. Si se le quitan dos o más, sigue siendo un libro. Pero si se le quitan todas las hojas, no es más un libro. ¿En qué momento ha dejado de ser libro? Percibir un libro, al igual que un perro, es un juicio perceptual que posee elementos generales inferidos abductivamente. En definitiva, la segunda

18 Los juicios perceptuales indican el carácter abductivo que existe en la percepción. La distinción entre "Juicio Perceptual" y "Percepto" adquiere tonalidades de un tecnicismo avanzado, que puede entenderse fácilmente si se siguen las consideraciones de Luisi (2006), a saber: "For this reason we should find also in perceptual phenomena a character of interpretation, a part that belongs to the order of signification (and this is the third category), but also a part that comes before interpretation, that has no generality; it's a pure reaction against the blow of the external object and it's almost forced upon us (the second category). In the Harvard Lectures the first aspect is called perceptual judgment, while the second one, the passive and blind one, is called percept". En este sentido, el juicio perceptual es la inferencia de carácter general que hay en la percepción (terceridad), en tanto que el percepto es el resultado de una reacción contra el objeto externo. En el fondo, la distinción entre ambas parece ser más un elemento de gradación en el continuo peirceano, que una delimitación entre la realidad del mundo y la subjetividad del sujeto cognoscente. Asi lo considera Corrington (1993, pág. 59) al indicar lo siguiente: "The 'percept', that is, the unitary element at the base of experience, is a vague something that is immediately given shape by a perceptual judgment". En este context entiendo tal immediately como el resultado de la aplicación de la tercera tesis cotaria, que indica la imposibilidad de distinguir entre el juicio perceptual y la inferencia abductiva.

19 De lo que se trata es de determinar si el mundo es un estado de cosas o son las cosas mismas. En este contexto, de la segunda tesis cotaria, es donde Peirce expone el ejemplo del dibujo de su padre de la pared de piedras, que era realmente una línea serpenteante. 
tesis cotaria establece que en la percepción, en tanto juicios perceptuales, existen elementos generales.

La tercera tesis cotaria es que la inferencia abductiva se matiza (shade) en juicios perceptuales sin una línea divisoria o demarcación entre ellas. La explicación de Peirce no es tan clara como las anteriores. Él indica que esta tercera tesis consiste en mirar los juicios perceptuales como formas extremas de inferencias abductivas, lo que significa que la percepción opera inferencialmente.

Peirce considera que de admitir la tercera tesis cotaria, habría que aceptar la segunda y por ende la primera. Se trata de establecer un marco lógico para la abducción, que por un lado limita con la forma lógica de la inferencia y por otro lado limita con el percepto. Pero como se ha visto, de lo que se tratan las tres tesis cotarias es exactamente de vincular, en un continuo, la percepción (juicio perceptual) y la abducción (inferencia). Es exactamente esta la configuración pragmatista (como forma de orientar la experiencia) de la abducción. La forma expresa de esta configuración está expresada en la conocida formulación peirceana: Los elementos de todo concepto entran en el pensamiento lógico por la puerta de la percepción y salen por la puerta de la acción deliberada; $y$ todo lo que no pueda mostrar su pasaporte en ambas puertas ha de ser detenido como no autorizado por la razón. ${ }^{20}$

Como se acaba de señalar, las tesis cotarias establecen el carácter abductivo (inferencial) de la percepción. La vinculación del pragmatismo a la abducción está sostenida sobre estas tres tesis cotarias, que en palabras de Peirce, le brindan al pragmatismo su carácter especial (EP, II, 16, PLA p. 226).

La pregunta por la novedad y la lógica del descubrimiento encuentran una especial respuesta en el repaso juicioso de las tesis cotarias. Cada tesis cotaria posee una implicación específica al pensar la novedad en la ciencia. La primera radica en la conservación de la primera tesis cotaria, la cual implica que en la abducción los conceptos y perceptos están en una relación inferencial. La ausencia de novedad se entiende en términos absolutos no relativos. Lo absolutamente nuevo resultaría completamente ininteligible, en la medida en que no podría establecerse ningún tipo de relación inferencial. Así, lo novedoso es tal en relación a un marco determinado

20 The elements of every concept enter into logical thought at the gate of perception and make their exit at the gate of purposive action; and whatever cannot show its passports at both those two gates is to be arrested as unauthorized by reason. (EP, II, 16, PLA p. 241) 
de referencia. Si puede formularse una figura geométrica novedosa, será nueva respecto a las relaciones espaciales de las figuras geométricas (ángulos, extensión, líneas, etc.). No puede tratarse de una figura geométrica que sea novedosa respecto a todo lo que haya en el mundo. De este modo, la novedad en la percepción posee el carácter sorpresivo que resulta de un determinado hecho; la percepción, como forma abductiva, busca resolver tal novedad a partir de la postulación de una abducción.

Un claro ejemplo para ilustrar esta implicación consiste en la percepción de un nuevo color. Ciertamente se percibe dentro de una generalidad cromática (como azul, rojo, verde, etc.) antes que como un tono específico. De tal manera, si nunca se hubiera visto un determinado tono, abductivamente se explica dentro del rango cromático de un cierto color, abducción de la cual pueden seguirse deducciones e inducciones y nuevas abducciones que perfilarán la comprensión de tal color.

Es con ello que la segunda tesis cotaria entra en juego. El hecho de que en los juicios perceptuales haya elementos generales, permiten que la novedad pueda resolverse por medio de una inferencia, puesto que aquí la novedad funcionaría dentro de una generalidad. Así, es posible comprender que la novedad obedece a un elemento no esperado, pero esperable. Es por eso que la comprensión milagrosa dentro de la abducción pragmatista resulta ininteligible. El caso que Peirce trabaja a este respecto es el de la transubstanciación, ${ }^{21}$ pero quizás resulte más ilustrativo el que Wittgenstein ofrece en la conferencia sobre ética (1989) cuando pone el ejemplo del hombre con cabeza de león.

Piensen en el caso de que a uno de ustedes le crezca una cabeza de león y empiece a rugir. Tan pronto como nos hubiéramos repuesto de la sorpresa, lo que yo sugeriría sería buscar un médico e investigar científicamente el caso y, si no fuera porque ello le produciría sufrimiento, le haría practicar una vivisección. ¿Dónde estaría entonces el milagro? Está claro que, en el momento en que miráramos las cosas así, todo lo milagroso habría desaparecido... La verdad es que el modo científico de ver un hecho no es el de verlo como un milagro. (Wittgenstein, 1989).

21 Pero para una postura sintética de esta consideración, remito al lector a (Corrington, 1993, pág. 38) en donde el autor refiere, siguiendo a Peirce, que la dificultad del dogma católico de la transubstanciación consiste en asumir un cambio en lo idéntico perceptiblemente, considerando un milagro en el cambio de la substancia. 
El ejemplo de Wittgenstein sirve para ilustrar el papel de la segunda tesis cotaria. Ciertamente la novedad en ella queda cobijada por una generalidad. Al hombre que le crece la cabeza de león tiene una biología y podría hacérsele una serie de estudios. La novedad no funciona como una especie de "milagro" en la ciencia. Lo que no ha sido explicado es aquello cuya novedad no logra agruparse inferencialmente dentro de la generalidad, lo que a su vez significa que la novedad persiste mientras no se logre resolver el carácter sorpresivo de un hecho. En el momento en que se explique porqué a alguien le ha crecido la cabeza de león, en ese momento el hecho deja de ser sorpresivo.

La anulación de lo absolutamente sorpresivo y novedoso, como un milagro, pone nuevamente de relieve la consecuencia de la primera tesis cotaria y que, como dijimos, consiste en entender que la novedad es relativa a un marco de referencia en el que resulta novedosa. Con las implicaciones de ambas tesis cotarias, la de la tercera puede verse como la consecuencia lógica de ambas.

La novedad, para la tercera tesis cotaria se resuelve en el orden de que toda novedad es fruto de una inferencia, razón por la cuál es imposible la búsqueda de un principio u origen (intuición) de la novedad por fuera del marco de las inferencias abductivas. Lo novedoso no podría traspasar el marco de los juicios perceptivos y de las inferencias en su continuo. De hacerlo no resultaría novedoso pues sería ininteligible. Por tanto, lo novedoso lo es respecto a una red de inferencias, que a su vez se sostienen en deducciones, inducciones, abducciones; esto es, sobre otras inferencias.

Por tanto, la contribución que el sistema de las tesis cotarias ofrece al pragmatismo como lógica de la abducción redunda fundamentalmente en las tareas que Peirce mismo les asigna: la fijación de la creencia como forma de resolver una duda genuina, lo que equivale a apaciguar el carácter sorpresivo de un hecho. La forma científica de fijar la creencia procede abductivamente y en este sentido adquiere una connotación de corrección, incluso dentro del marco de la comunidad. Por otro lado, la claridad de las ideas, como forma de resolver el carácter sorpresivo de un hecho obedece a la tarea del pragmatismo de rastrear el continuo de las abducciones, sin la posibilidad de identificar un punto cero de una idea clara y distinta o de una intuición, lo que equivaldría a la formulación de algo completamente nuevo, y por tanto incomprensible. 


\title{
3. Conclusiones
}

El análisis de las tesis cotarias y de su relación con la peculiar forma que le brindan al pragmatismo de Peirce ha llevado a la presente investigación a considerar que la novedad presente en la abducción es una novedad relativa, la novedad absoluta resulta ininteligible. De tal manera, si una novedad fuese puramente novedosa rompería la tercera tesis cotaria ya que sería tanto imposible de percibir como imposible de inteligir. En términos de este pragmatismo, una novedad tal resultaría incognoscible al no poder transitar del sensu al intellectu; y lógicamente resultaría ser una contradicción:

\begin{abstract}
En consecuencia, no-cognoscible, si se trata de un concepto, es un concepto de la forma "A, no A" y es, al menos, contradictorio en sí mismo. De este modo, la ignorancia y el error solo pueden concebirse como correlativos a un conocimiento y verdad reales, siendo estos últimos de la naturaleza de las cogniciones. Frente a toda cognición, existe una realidad desconocida pero cognoscible, pero frente a toda cognición posible, solo existe lo contradictorio en sí mismo. ${ }^{22}$
\end{abstract}

El carácter cognoscible de la novedad consiste en la posibilidad de ser comprendida y explicada mediante la abducción. De tal manera que lo sorpresivo/novedoso de un hecho solo lo es dentro de una generalidad que permite la formulación de inferencias que lo expliquen y aplaquen. En consecuencia, la novedad obedece a un juicio perceptual/percepto o al continuo de inferencias. Esto es, la novedad solo resulta comprensible dentro de la tercera tesis cotaria, que como se ha indicado, implica las otras dos.

Así, lo que se gana con la inclusión de las tesis cotarias en esta cuestión es la novedad dentro de la abducción puede resumirse en dos premisas:

La primera, la abducción explica por sí misma, pero no con independencia de las otras formas de inferencia, la novedad a partir de la generalidad dentro de la que se imbrica (terceridad, que queda señalada a partir de la segunda tesis cotaria). Esta formulación permite superar las dos primeras consideraciones acerca de la pregunta

22 Hence, not-cognizable, if a concept, is a concept of the form "A, not-A" and is, at least, self-contradictory. Thus, ignorance and error can only be conceived as correlative to a real knowledge and truth, which latter are of the nature of cognitions. Over against all possible cognition, there is an unknown but knowable reality. (EP, I, QCCF p. 25) 
por la novedad en la abducción, aquellas que indican la poca utilidad de la abducción para explicar la novedad (Soto, 2011) o quienes postulan una especie de "instinto" para guiar la abducción. En este sentido, es completamente relevante señalar que Peirce no niega que ciertos procesos abductivos puedan ser observados en animales $y$ en general en "seres autoconfigurados", pero estos han de entenderse como formas de pensamiento: El pensamiento no está conectado necesariamente con el cerebro. Este aparece en el trabajo de las abejas, de los cristales y a lo largo del puro mundo físico. ${ }^{23} \mathrm{La}$ consecuencia de esta formulación es que Peirce no habla de la abducción bajo un proceso psicológico de cómo la mente estipula una abducción, sino que refiere a la lógica que define las estructuras mismas del mundo y cuya comprensión resulta abductiva:

Peirce vincula el avance de la ciencia con el avance de la lógica. Cada paso en la evolución científica es también un paso en el entendimiento de las reglas de la inferencia. Peirce aclara que la lógica no es una disciplina que describe los hábitos de la vida mental, sino una disciplina que describe las reglas por las cuales la vida mental debe ser gobernada... Peirce no deriva la lógica de la psicología humana, sino simplemente parece ir en el otro extremo, derivando de los principios de la lógica la psicología humana. ${ }^{24}$

La segunda proposición afirma que el pragmatismo peirceano, como lógica de la abducción, establece una orientación de la experiencia mediante la formulación de inferencias acerca de la realidad que permiten generar y proteger nuevas reglas para la acción. (Corrington, 1993, p. 37). Estas reglas consisten en la fijación de las creencias y la aclaración de las ideas que, como se ha indicado, y el mismo Peirce lo enfatiza, constituyen las tareas fundamentales del pragmatismo. (EP, II, PLA p. 239). La experiencia adquiere una guía mediante la abducción ya que define el ámbito de lo

23 Traducción propia: "Thought is not necessarily connected with a brain. It appears in the work of bees, of crystals, and throughout the pure physical world..." (CP 4.551) Esta es la tesis central, aplicada a las formas de auto-organización (Gonzalez \& Haselager, 2005): "In summary, the central hypothesis of the present paper is that creativity is a self-organizing process in which abductive reasoning occurs allowing the expansion of well- structured belief."

24 Traducción propia: Peirce links the advance of science with the advance of logic. Each step in scientific evolution is also a step in our understanding of the rules of inference. Peirce makes it clear that logic is not a discipline describing the habits of mental life, but a discipline describing the rules by which mental life should be governed... Peirce does not derive logic from human psychology, but sometimes appears to go in the other extreme of deriving human psychology from the principles of logic. (Corrington, 1993, pág. 30) 
expectable y deja abierta la posibilidad de comprensiones ante lo también posible pero inesperado, o sorpresivo. Por tanto, la forma abductiva, que es falible y susceptible de corrección, permite establecer reglas de acción.

La vinculación entre hábito y regla de acción es fundamental en el pragmatismo, ya que define la manera en que se orienta la experiencia. Este es el sentido profundo de la concepción peirceana de la creencia ${ }^{25}$ y a partir de esta puede considerarse que el pragmatismo cumple con su tarea básica de asegurar la experiencia en el mundo a partir de abducciones 'corregibles' desde la fijación de la creencia. Así, puede considerarse que el pragmatismo peirceano está comprometido con aumentar la inteligibilidad y la generalidad en el mundo.

\section{Referencias}

\section{Fuentes principales}

Los textos citados de Peirce corresponden a dos fuentes. La primera es Essential Peirce Vol. I y II, Indiana University Press, 1992. Las citas se referencian con las siglas EP, sigue el número del volumen, las iniciales del texto en mayúsculas y la página. Las referencias que inician con las siglas CP corresponden a la versión de los Collected Papers.

Se han seguido las siguientes convenciones:

DIH - Deducción, inducción, hipótesis

PLA - Pragmatismo como lógica de la abducción (Pragmatismo y abducción)

FI - Algunas consecuencias de cuatro incapacidades

NM - La Naturaleza del Significado

QCCF - Cuestiones de ciertas facultades atribuidas al hombre

25 [belief has three properties]... and third, it involves the establishment in our nature of a rule of action, or say for short, a habit. (EP, 1 HMIC p. 129) 


\section{Fuentes secundarias}

Callaway, H. G. (2008). Abduction, pragmatism and the scientific imagination. Conference of the Wesleyan Philosophical Society. Durham.

Corrington, R. (1993). An introduction to C.S. Peirce. Boston: Rowman \& Littlefield Publishers.

Génova, G. (1997). Charles Peirce: La lógica del descubrimiento. CUADERNO DE ANUARIO FILOSOFICO No. 45.

Gonzalez, M., \& Haselager, W. (2005). Creativity: Surprise and abductive reasoning. Semiotica, 325-341.

Gorlée, D. (1996). ¡Eureka! La traducción como un descubrimiento pragmático. From Sitio Web del Grupo de Estudios Peirceanos - Universidad de Navarra: http:// www.unav.es/gep/AF/Gorlee.htm

Luisi, M. (2006). Percept and perceptual judgment in Peirce's phenomenology. Cognitio-Estudos: Revista eletrônica de Filosofia.

Nubiola, J. (2005). Abduction or the logic of surprise. Semiótica , 117-130.

Paavola, S. (En prensa). Peircean abduction: instinct of inference. Semiotica, Por Definir.

Soto, C. (2006). Abducción como 'inferencia’ y como 'intuición’ en Peirce. Octava Versión de las Jornadas de Lógica y Filosofía de las Ciencias. Santiago de Chile: Universidad de Santiago de Chile.

(2011). Peirce, la abducción y el problema del descubrimiento en filosofía de la ciencia. CADERNOS UFS FILOSOFIA, 95-112.

Wittgenstein, L. (1989). Conferencia sobre ética. Madrid: Paidos. 